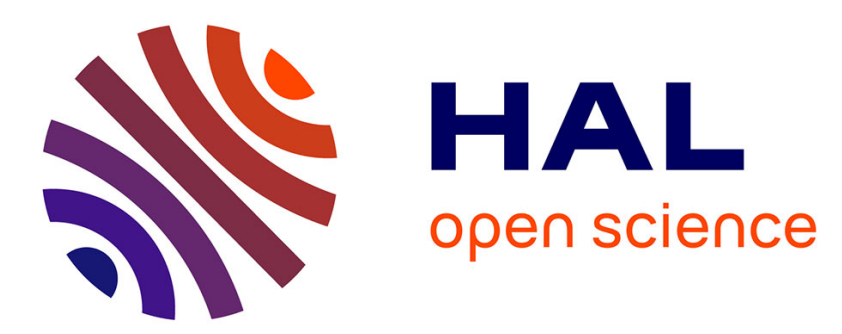

\title{
PRÉDICTION DU RAYONNEMENT ACOUSTIQUE D'UNE PLAQUE TROUÉE
}

O. Beslin, Patrick Millot, Jean-Louis Guyader

\section{To cite this version:}

O. Beslin, Patrick Millot, Jean-Louis Guyader. PRÉDICTION DU RAYONNEMENT ACOUSTIQUE D'UNE PLAQUE TROUÉE. Journal de Physique IV Proceedings, 1992, 02 (C1), pp.C1-503-C1-506. 10.1051/jp4:19921108 . jpa-00251062

\section{HAL Id: jpa-00251062 https://hal.science/jpa-00251062}

Submitted on 1 Jan 1992

HAL is a multi-disciplinary open access archive for the deposit and dissemination of scientific research documents, whether they are published or not. The documents may come from teaching and research institutions in France or abroad, or from public or private research centers.
L'archive ouverte pluridisciplinaire HAL, est destinée au dépôt et à la diffusion de documents scientifiques de niveau recherche, publiés ou non, émanant des établissements d'enseignement et de recherche français ou étrangers, des laboratoires publics ou privés. 


\title{
PRÉdiction DU RAYONNEMENT ACOUSTIQUE D'UNE PLAQUE TROUEE
}

\author{
O. BESLIN, P. MILLOT et J.L. GUYADER* \\ Laboratoire des Sciences de l'Habitat, Ecole Nationale des Travaux Publics de l'Etat, \\ F-69518 Vaulx-en-Velin, France \\ "Laboratoire Acoustique et Vibrations, Institut National des Sciences Appliquées, \\ F-69621 Villeurbanne, France
}

\begin{abstract}
Sound radiation from plates with holes is of practical interest in the design of machinery hoods but also in that of double walls with one perforated panel (ref. $\{1\})$. A theoretical model is established to calculate the pressure radiated in two rectangular rooms separated by a perforated plate. An integral formulation is used to resolve the acoustical field and a variational method is used to resolve the vibrational field. First numerical results are shown in a last part.
\end{abstract}

\section{INTRODUCTION.}

Nous traitons le cas d'un local parrallélépipédique de dimension $\mathrm{L}_{\mathrm{x}} \times \mathrm{L}_{\mathrm{y}} \times \mathrm{L}_{\mathrm{z}}$, séparé en deux à la cote $z=z_{p}$ par une plaque trouée de dimension $L_{x} \times L_{y}$. Nous appelons $p(x, y, z)$ la pression acoustique dans le local et $w(x, y)$ la déformée de la plaque supposée vibrer en flexion avec des conditions aux limites "simplement appuyée". Le système ainsi constitué est étudié en terme de rayonnement acoustique d'une plaque troué (réponse à une excitation mécanique de la plaque par une densité surfacique de force : $\bar{F}(x, y))$ et en terme de tranparence acoustique d'une plaque trouée (réponse à une excitation acoustique par une surface $S_{a}$ vibrant en piston à la vitesse $V_{\omega}$ dans le plan $z=0$ ).

La présence de la plaque en $z=z_{p}$ génère un saut de pression dans le local :

$$
\bar{P}(x, y)=P\left(x, y, z_{p}^{+}\right)-P\left(x, y, z_{p}\right)
$$

Les inconnues du système à résoudre sont :

- La déformée de la plaque que nous appelons $W(x, y)$

- Le saut de pression $\bar{P}(x, y)$ que nous chercherons sous la forme

$$
\overline{\mathrm{P}}(\mathrm{x}, \mathrm{y})=\sum_{\mathrm{r}, \mathrm{s}} \overline{\mathrm{P}}_{\mathrm{rs}} \cos \left(\frac{\mathrm{r \pi x}}{\mathrm{L}_{\mathrm{x}}}\right) \cos \left(\frac{\mathrm{r} \pi \mathrm{y}}{\mathrm{L}_{\mathrm{y}}}\right) \quad(\mathrm{r}, \mathrm{s} \text { variant de } 0 \mathrm{a} \infty)
$$

Dans une première partie nous décrivons l'opérateur de plaque $Z_{p}$, reliant la déformée $W(x, y)$ au saut de pression $\overline{\mathbf{P}}(\mathrm{x}, \mathrm{y})$ et à l'excitation mécanique $\overline{\mathbf{F}}(\mathbf{x}, \mathbf{y})$ :

$$
Z_{p}(W(x, y))=\bar{F}(x, y)-\bar{P}(x, y)
$$

Dans une deuxième partie, nous décrivons le champ de pression généré par le saut de pression $\overline{\mathrm{P}}(\mathrm{x}, \mathrm{y})$ en $z=z_{p}$ et la surface vibrante $S_{a}$ en $z=0$ puis via l'équation d'Euler, nous en déduisons la composante selon $z$ de la vitesse particulaire en $z=z_{p}: V_{z}\left(x, y, z_{p}\right)$ ainsi, une seconde relation (IV) traduisant la continuité des vitesse normales acoustiques et de plaque vient compléter la relation (III) pour décrire totalement l'équilibre du système et en sortir les inconnues $W(x, y)$ et $\bar{P}(x, y)$ :

$$
V_{z}\left(x, y, z_{p}\right)=j \omega W(x, y)
$$


Il est possible ensuite à partir de $\mathrm{W}(\mathrm{x}, \mathrm{y})$ et $\overline{\mathrm{P}}(\mathrm{x}, \mathrm{y})$ de reconstruire toutes les variables du problème.

Enfin, dans une troisième partie, nous présentons les résultats de la déformée d'une plaque et du saut de pression qu'elle génère en réponse à une excitation acoustique.

\section{L'OPERATEUR DE PLAOUE}

Pour décrire l'opérateur de plaque, nous utilisons une approche variationnelle, en utilisant comme fonctions de bases, les fonctions propres d'un operateur de plaque pleine: $\left\{\phi_{\mathrm{nm}}\right\}$ Ainsi, nous cherchons la déformée d'une plaque trouée sous la forme :

$$
W(x, y)=\sum_{n, m=1}^{\infty} b_{n m} \cdot \phi_{n m} \quad \text { où } \quad \phi_{n m}=\sin \left(\frac{n \pi x}{L x}\right) \cdot \sin \left(\frac{m \pi y}{L y}\right)
$$

Pour assurer l'unicité de la décomposition de $W(x, y)$ dans la base $\left\{\phi_{\mathrm{nm}}\right\}$, nous sommes contraints de définir sur tout le domaine $S_{\mathrm{T}}=\mathrm{L}_{\mathrm{x}} \times \mathrm{L}_{\mathrm{y}}$ des caractéristiques mécaniques (y compris sur la surface des trous $S_{t}$ ).

Ainsi nous définissons sur la surface de la plaque $S_{p}$ une raideur $: D=\frac{E h^{3}}{12\left(1-v^{2}\right)}$ et une densité surfacique $M=p . h$. De même, nous définissons sur là surface des trous : $S_{t}$ une raideur $\widetilde{D}=\varepsilon_{d} D$ et une densité surfacique $: \widetilde{M}=\varepsilon_{m} M$ dite "ectoplasmique", où $\varepsilon_{d}$ et $\varepsilon_{m}$ sont très petits devant l'unite ( $\sim 10^{-7}$ à 10-15). Les caractéristiques mécaniques des trous sont négligeables devant celles du milieu acoustique, mais non négligeables devant les erreurs numériques d'arrondis.

En résumé, la plaque trouée est introduite comme une plaque inhomogène dont les caractéristiques mécaniques sont:

$$
\left.\left.\begin{array}{c}
M(x, y)=M \\
D(x, y)=D
\end{array}\right\} \operatorname{Si~}(x, y) \in S_{p} \quad \begin{array}{c}
M(x, y)=\varepsilon_{m} \cdot M \\
D(x, y)=\varepsilon_{d} \cdot D
\end{array}\right\} \text { Si }(x, y) \in S_{t}
$$

Le Hamiltonien d'une telle plaque s'écrit :

$$
\begin{aligned}
H=\int_{q} \int^{2} & \int_{s_{T}} \frac{M(x, y)}{2} \cdot(\dot{W}(x, y))^{2} \\
& -\frac{D(x, y)}{2} \cdot\left(\left(\partial_{x x}^{2} W(x, y)\right)^{2}+\left(\partial_{y y}^{2} W(x, y)\right)^{2}+2 \cdot \vartheta \cdot \partial_{x x}^{2} W(x, y) \cdot \partial_{y y}^{2} W(x, y)+2 \cdot(1-\vartheta) \cdot\left(\partial_{x y}^{2} W(x, y)\right)^{2}\right\} \\
& +(\bar{F}(x, y)-\bar{p}(x, y)) \cdot W(x, y) \cdot d s \cdot d t
\end{aligned}
$$

L'extrémisation de $\mathrm{H}$ en regard des coefficients $b_{\mathrm{nm}}$ nous conduit au système suivant :

$$
\sum_{n, m} Z_{n m p q} \cdot b_{n m}=\bar{F}_{p q}-\sum_{r, s} C_{r s} S_{p q} \cdot \bar{p}_{r s}
$$

où $\mathrm{Z}_{\mathrm{nmpq}}$ sont les eléments de matrice représentant l'operateur $\mathrm{Z}$ dans la base des $\left\{\phi_{\mathrm{nm}}\right\}, \overline{\mathrm{F}}_{\mathrm{pq}}$ les forces généralisées, $\mathrm{C}_{\mathrm{rs}} \mathrm{S}_{\mathrm{pq}}$ une matrice de changement de base. Une validation en terme de modes propres de cet operateur à déjà été presentée (ref. $\{2\},\{3\})$. Une confrontation avec une methode élément fini sur les 10 premier modes d'une plaque trouée est donnée en figure 1

\section{EOUATION DE CONTINUITE DES VITESSES}

Le local considéré constitue un volume $V$ enveloppé par une surface fermée $\Sigma$ : en utilisant une formulation intégrale, la pression acoustique au point $\mathbf{M}(\mathrm{x}, \mathrm{y}, \mathrm{z})$ peut être décrite en fonction d'une fonction de $G r e e n G_{k}\left(M, M_{0}\right)$ du local et des termes de sources monopolaires (surface vibrante $S_{a}$ en $z=0$ de vitesse $V_{\omega}$ ) et dipolaire (saut de pression à la traversée de la plaque).

$$
p(M)=\int_{s}^{j} j \cdot \omega \cdot \rho_{a} \cdot V_{\omega} \cdot G_{k}\left(M, M_{0}\right) \cdot d S_{0}+\int_{S_{T}} \bar{p}\left(x_{0}, y_{0}\right) \cdot \partial_{z_{0}} G_{k}\left(M, M_{0}\right) \cdot d S_{0}
$$

$\mathrm{M}_{0}\left(\mathrm{x}_{0}, \mathrm{y}_{0}, \mathrm{z}_{0}\right)$ coordonnées des points sources., $\rho_{\mathrm{a}}$ densité de l'air 
$\mathrm{G}_{\mathbf{k}}$ telle que :

$$
\left\{\begin{array}{ll}
\left(\Delta+k^{2}\right) G_{k}\left(M, M_{0}\right)=0 & M \in V \\
\partial n_{0} G_{k}\left(M, M_{0}\right)=0 & M \in \Sigma
\end{array} \quad k^{2}=\frac{\omega^{2}}{c_{0}^{2}} \frac{1}{1+j \eta}\right.
$$

$\overrightarrow{\mathrm{n}_{0}}$ normale extérieure à $\Sigma$

Compte tenu de la geométrie du problème, nous pouvons développer $\mathrm{G}_{\mathbf{k}}\left(\mathrm{M}, \mathrm{M}_{0}\right)$ sur deux indices, comme le suggère M. BRUNEAU (ref. $\{4\}$ ):

nous trouvons :

$$
\mathrm{G}_{\mathrm{k}}\left(\mathrm{M}, \mathrm{M}_{0}\right)=\sum_{\mathrm{r}, \mathrm{s}} \mathrm{g}_{\mathrm{rs}}\left(\mathrm{z}, \mathrm{z}_{0}\right) \cdot \cos \left(\frac{\mathrm{r} \cdot \pi \cdot \mathrm{x}}{\mathrm{Lx}}\right) \cdot \cos \left(\frac{\mathrm{s} \cdot \pi \cdot \mathrm{y}}{\mathrm{Ly}}\right) \cdot \cos \left(\frac{\mathrm{r} \cdot \pi \cdot \mathrm{x}_{0}}{\mathrm{Lx}}\right) \cdot \cos \left(\frac{\mathrm{s} \cdot \pi \cdot \mathrm{y}_{0}}{\mathrm{Ly}}\right)
$$

$$
\begin{aligned}
& \mathrm{g}_{\mathrm{rs}}\left(\mathrm{z}, \mathrm{z}_{0}\right)=\frac{-1}{\mathrm{~N}_{\mathrm{rs}} \cdot \mathrm{k}_{\mathrm{zrs}} \cdot \sin \left(\mathrm{k}_{\mathrm{zrs}} \cdot \mathrm{L}_{\mathrm{z}}\right)} \times \cos \left(\mathrm{k}_{\mathrm{zrs}} \cdot\left(\mathrm{L}_{\mathrm{z}}-\mathrm{z}_{0}\right)\right) \cdot \cos \left(\mathrm{k}_{\mathrm{zrs}} \cdot \mathrm{z}\right) \quad \text { si z }\left\langle\mathrm{z}_{0}\right. \\
& \left.\mathrm{g}_{\mathrm{rs}}\left(\mathrm{z}, \mathrm{z}_{0}\right)=\frac{-1}{\mathrm{~N}_{\mathrm{rs}} \cdot \mathrm{k}_{\mathrm{zrs}} \cdot \sin \left(\mathrm{k}_{\mathrm{zrs}} \cdot \mathrm{L}_{\mathrm{z}}\right)} \times \cos \left(\mathrm{k}_{\mathrm{zrs}} \cdot\left(\mathrm{L}_{\mathrm{z}}-\mathrm{z}\right)\right) \cdot \cos \left(\mathrm{k}_{\mathrm{zrs}} \cdot \mathrm{z}_{0}\right) \text { si } \mathrm{z}\right) \mathrm{z}_{0} \\
& \text { où } k_{z r s}^{2}+\left(\frac{r . \pi}{L_{x}}\right)^{2}+\left(\frac{s . \pi}{L_{y}}\right)^{2}=k^{2} \quad \text { et } N_{r s}=\frac{L_{x} \cdot L_{y}}{4} \cdot\left(1+\delta_{0, r}\right) \cdot\left(1+\delta_{0, s}\right)
\end{aligned}
$$

En reportant la fonction de Green dans l'expression de la pression donnée en (VI) et en utilisant l'équation d'Euler, nous pouvons établir l'équation de continuité des vitesses :

$$
\frac{\sin \left(k_{z r s}\left(z_{p}-L_{z}\right)\right)}{\sin \left(k_{z r s} \cdot L_{z}\right)} \cdot V_{\omega r s}+\frac{N_{r s} \cdot k_{z r s}}{j \cdot \omega \cdot \rho_{a}} \cdot \frac{\sin \left(k_{z r s}\left(z_{p}-L_{z}\right)\right) \cdot \sin \left(k_{z r s} \cdot z_{p}\right)}{\sin \left(k_{z r s} \cdot L_{z}\right)} \cdot \bar{p}_{r s}=\sum_{n, m} C_{r s} s_{n m} \cdot j \cdot \omega \cdot b_{n m}
$$

Terme dû à la source $S_{\mathrm{a}}$ Terme dû à la présence de la plaque Vitesse acoustique selon $z$ en $z=z_{p}$ $=$ vitesse de plaque

La resolution du système constitué des équations (VI) et (VIII) nous permet alors d'identifier les inconnues $\overline{\mathrm{p}}_{\mathrm{rs}}$ et $\mathrm{b}_{\mathrm{nm}}$

\section{EXEMPLE DE RESOLUTION:}

Nous présentons en figure 2 le cas d'un local de dimensions: $\mathrm{Lx}=1 . \mathrm{m}, \mathrm{Ly}=0.7 \mathrm{~m}, \mathrm{Lz}=2 . \mathrm{m}$, zp $=0.5 \mathrm{~m}$ séparé par une plaque d'acier de $3 . \mathrm{mm}$ possédant un trou rectangulaire de $0.30 \mathrm{~m}$ par $0.35 \mathrm{~m}$, et excité par une source acoustique à la frequence $500 \mathrm{~Hz}$. La figure 2a represente la déformée de la plaque: On notera les grandes amplitudes dans le trou relativement à la surface $S p$. La figure $2 \mathrm{~b}$ represente le saut de pression: Nous voyons qu'il tend à s'annuler dans le trou comme il se doit. Ces résultats ont été obtenus en tronquant les séries en $(n, m)$ à 645 termes, et les séries en $(r, s)$ à 282 termes

\begin{tabular}{|ll|ll|}
\hline \multicolumn{2}{|c|}{ variational } & \multicolumn{2}{|c|}{ F.E.M. } \\
\hline 6.53 & $\mathrm{~Hz}$ & 6.43 & $\mathrm{~Hz}$ \\
\hline 18.7 & $\mathrm{~Hz}$ & 18.6 & $\mathrm{~Hz}$ \\
\hline 20.6 & $\mathrm{~Hz}$ & 20.5 & $\mathrm{~Hz}$ \\
\hline 31.0 & $\mathrm{~Hz}$ & 31.0 & $\mathrm{~Hz}$ \\
\hline 37.4 & $\mathrm{~Hz}$ & 37.3 & $\mathrm{~Hz}$ \\
\hline 40.8 & $\mathrm{~Hz}$ & 40.9 & $\mathrm{~Hz}$ \\
\hline 47.0 & $\mathrm{~Hz}$ & 46.9 & $\mathrm{~Hz}$ \\
\hline 56.9 & $\mathrm{~Hz}$ & 57.1 & $\mathrm{~Hz}$ \\
\hline 61.1 & $\mathrm{~Hz}$ & 61.4 & $\mathrm{~Hz}$ \\
\hline 7.9 .6 & $\mathrm{~Hz}$ & 68.0 & \\
\hline
\end{tabular}

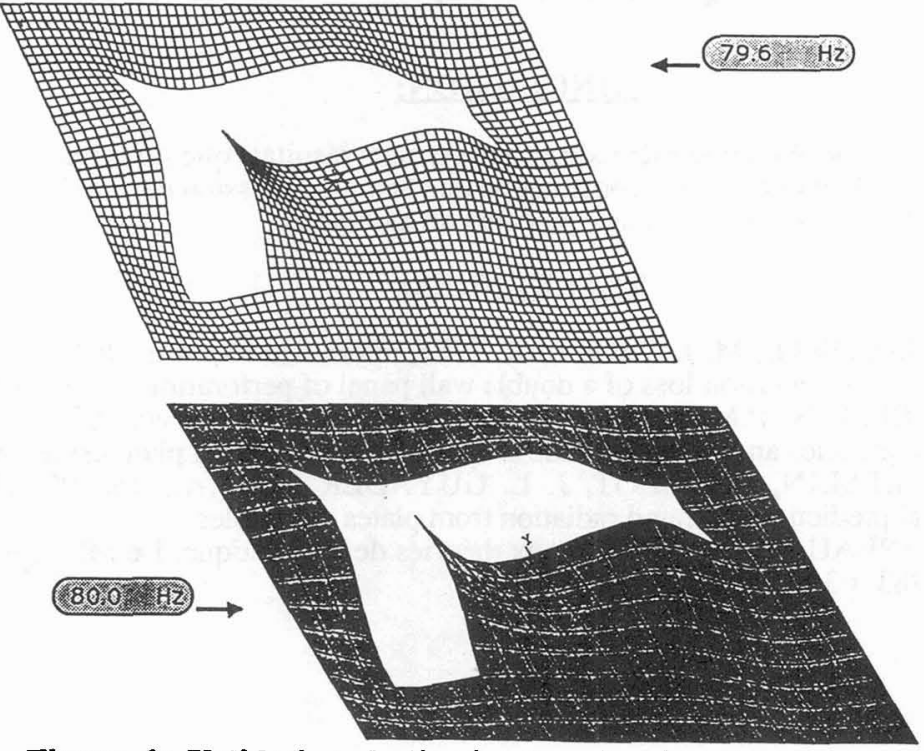

Figure 1: Validation de l'opérateur de plaque 


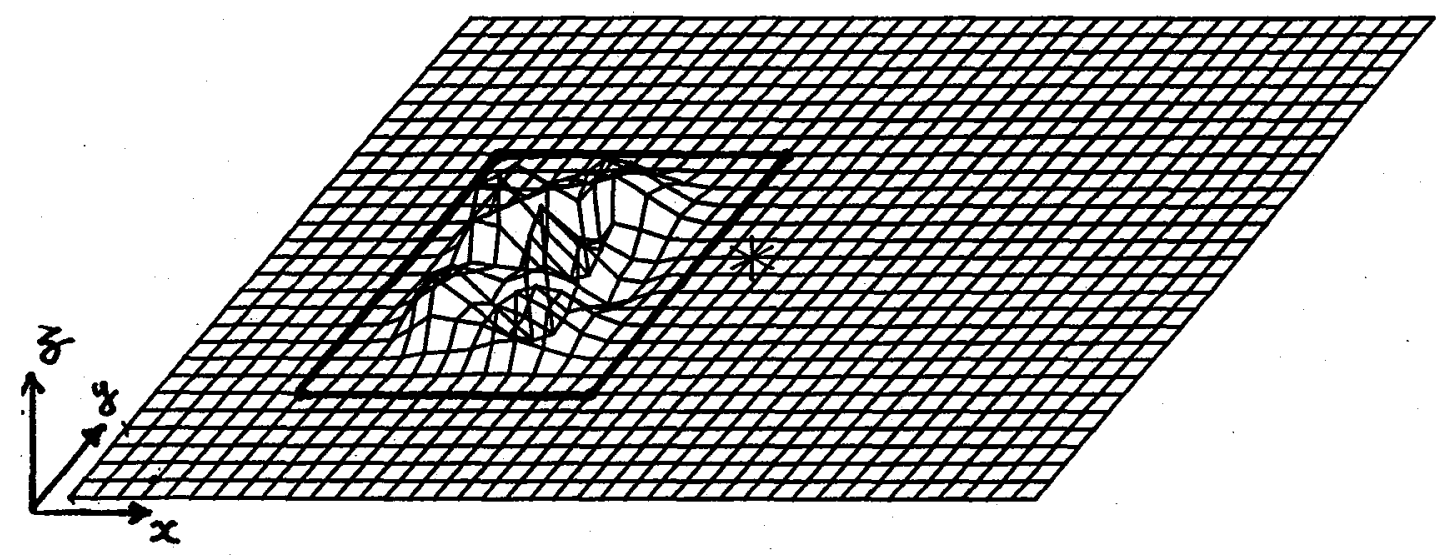

Figure 2a: deformée de la plaque trouée (contour du trou en gras)

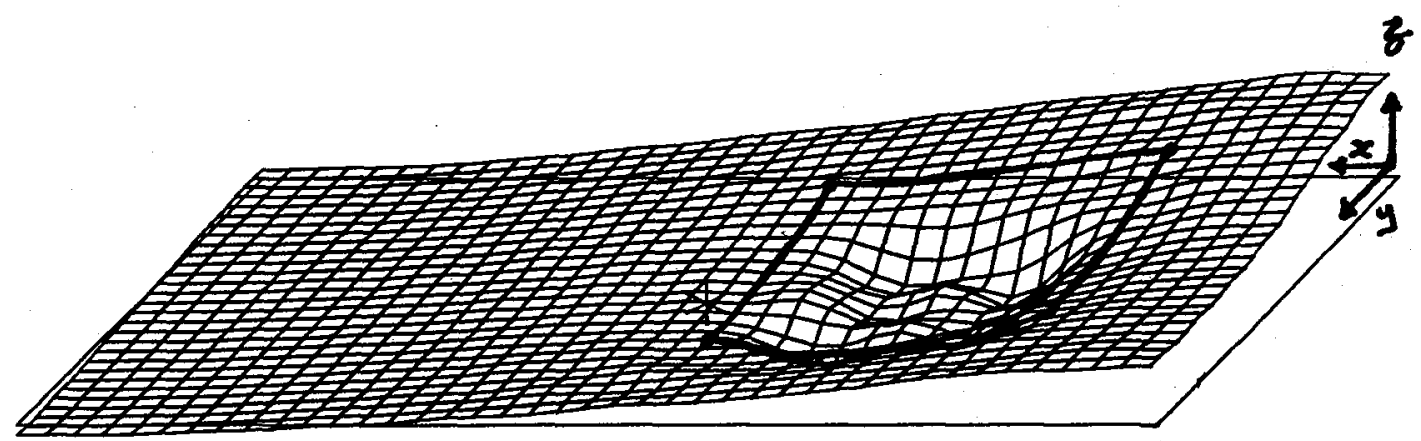

Figure 2b: saut de pression à la traversée de la plaque.

\section{CONCLUSION:}

Nous avons exposé ici une méthode et les premiers résultats que nous pouvons en attendre. Cette étude se poursuit actuellement par la reconstruction du champ de pression dans le local et les résultats de simulation seront confrontés à une expérience en cours de montage.

\section{REFERENCES:}

11) M. S. ATWAL, M. J. CROCKER, 1985, internoise 85, Munich 18-20 sept., 1985, p.389-391. The effect on the transmission loss of a double wall panel of perforating the second panel.

(2) O. BESLIN, P.MLLOT, J. L. GUYADER, J.A.S.A., vol. 89, N0. 4, Pt. 2, 2 SA10 (1991), , Natural frequencies and bending mode shapes of thin perforated plate using a variational method.

(3) O. BESLIN, P.MILLOT, J. L. GUYADER, J.A.S.A., vol. 90, NO. 4, Pt. 2, 2SA3 (1991), Theoretical prediction of sound radiation from plates with holes.

(4) BRUNEAU M. Introduction aux théries de l'acoustique. Le Mans, publication de l'Université du Maine. 1983, 634p, p.9-9-1 à 9-9-4 